(C) 1984. The Genetical Society of Great Britain

\title{
A POSSIBLE EXPLANATION OF CYCLICAL PARTHENOGENESIS
}

\author{
ALEXEY S. KONDRASHOV \\ Research Computer Centre, Academy of Sciences of the USSR 142292, Pushchino, Moscow \\ Region, USSR
}

Received 11.vii. 83

Factors that compensate for the two-fold advantage of parthenogenesis and that may account for the maintenance of sexual reproduction have been widely discussed (Maynard Smith, 1978). We have stated elsewhere (Kondrashov, 1982) that sexual reproduction is advantageous if only those individuals with less than some critical $(K)$ number of deleterious mutations are viable. The numerous examples of stable co-existence of sexual and parthenogenetic methods of reproduction provide good evidence for a short-term advantage of sex. One of these strategies, namely, cyclical parthenogenesis, can be readily explained in terms of selection against deleterious mutations by assuming that their critical number, $K$, changes periodically.

There are many animals, such as water fleas, rotifers and aphids, that are apomictic parthenogens under favourable conditions (usually in summertime); however, when conditions deteriorate (in the autumn) they make use of sex to produce winter eggs. We may assume that a winter egg needs to have no more than $K_{1}$ mutations to outlive the unfavourable winter period and produce a parthenogenetic clone in spring. Then, under favourable conditions a viable individual may not have more than $K_{2}$ mutations; reasonably $K_{1}<K_{2}$. Let the average and the variance of the number of mutations per individual in a population surviving the winter be $m_{1}$ and $V_{1}$, respectively. Evidently, $m_{1}<K_{1}$ and $V_{1}<m_{1}$ because of truncation selection. In the spring the critical mutation number grows to equal $K_{2}$, and the number of mutations per individual becomes far less than the new critical number. Consequently, selection against them does not work for a time and the two-fold advantage of parthenogenesis plays the decisive part (fig. 1). Accumulation of deleterious mutations during the summer will result in an increase of the average and the variance of the number of mutations per individual of $m_{2}$ and $V_{2}$. As the number of new mutations per genome has a Poisson distribution, $m_{2}=V_{2}$. Then in autumn the average and the variance of the number of mutations would be $m=m_{1}+m_{2}$ and $V=V_{1}+V_{2}$. Under a sufficiently large mutation rate $m>K_{1}$. If $K_{1}$ is large enough $V$, can be considerably smaller than $m$. If the mutation rate is high enough for the truncation selection to start working in the summer, then the difference between $m$ and $V$ will be even larger.

In contrast, even one sexual generation makes the distribution of mutations at different loci almost independent, resulting in the growth of $V$ to equal the value of $m$. Note that even a moderate growth of $V$, before $K_{1}$ substitutes $K_{2}$, would lead to a substantial increase in the viability of the progeny (fig. 2). Therefore, when the mutation rate per genome, $K_{1}$, and $K_{2}-K_{1}$ are all sufficiently large, cyclical parthenogenesis is likely to develop. 


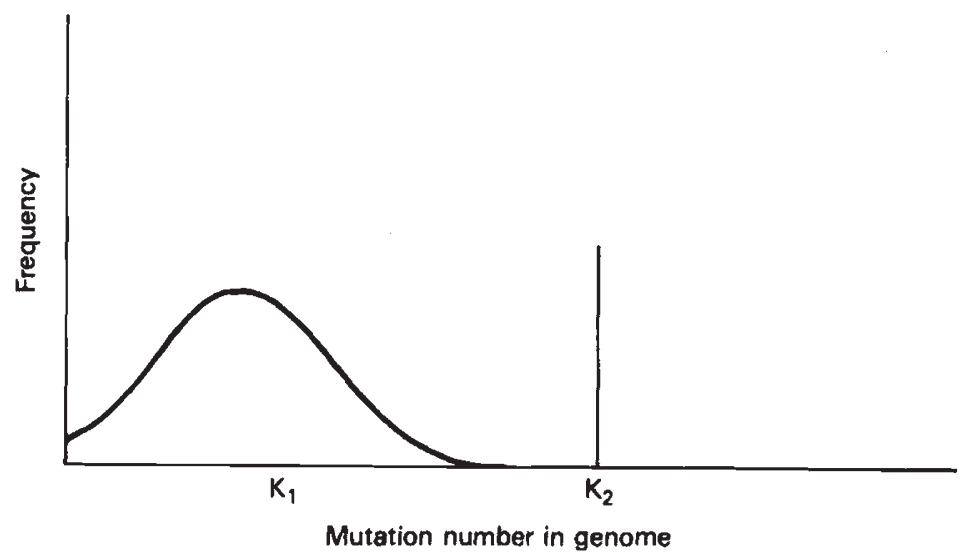

FIG. 1. Genotype frequencies at the beginning of summer, after substitution $K_{1} \rightarrow K_{2}$. All individuals carry less than the new critical number of mutations.

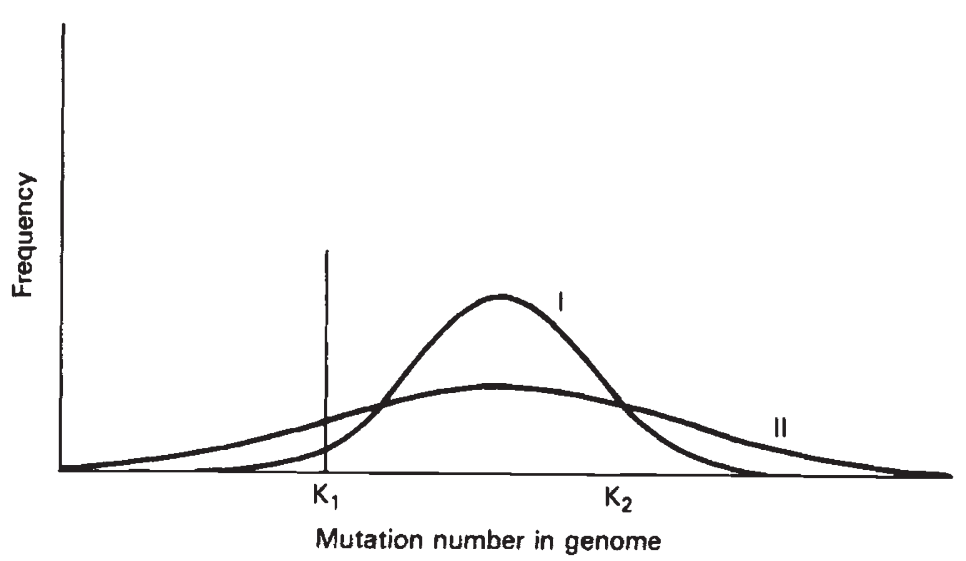

FIG. 2. Genotype frequencies in asexual (I) and sexual (II) progeny in autumn. The proportion of sexual progeny to survive winter is remarkably higher.

Alternation of favourable and unfavourable conditions may be irregular and vegetative reproduction may take the place of parthenogenesis, as with some fungi. In addition, with some green algae the form that survives unfavourable conditions is the only diploid stage of their life cycle, which is natural in view of the fact that the vast proportion of deleterious mutations are recessive. Consideration of deleterious mutations also suggests a plausible explanation of simultaneous co-existence of sexual and asexual reproduction, i.e., facultative parthenogenesis (to appear).

\section{REFERENCES}

MAYNARD SMITH, J. 1978. The Evolution of Sex. Cambridge University Press, Cambridge. KONDRASHOV, A. S. 1982. Selection against harmful mutations in large sexual and asexual populations. Genetical Research 40, 325-332. 